\title{
Strong approximation and a central limit theorem for St. Petersburg sums
}

\author{
I. Berkes *
}

\begin{abstract}
The St. Petersburg paradox (Bernoulli 1738) concerns the fair entry fee in a game where the winnings are distributed as $P\left(X=2^{k}\right)=2^{-k}, k=1,2, \ldots$ The tails of $X$ are not regularly varying and the sequence $S_{n}$ of accumulated gains has a remarkable asymptotic behavior: as Martin-Löf (1985) and Csörgő and Dodunekova (1991) showed, $S_{n} / n-\log _{2} n$ has a class of semistable laws as subsequential limit distributions. This has led to a clarification of the paradox and an interesting and unusual asymptotic theory in past decades. In this paper we prove that $S_{n}$ can be approximated by a semistable Lévy process $\{L(n), n \geq 1\}$ with a.s. error $O\left(\sqrt{n}(\log n)^{1+\varepsilon}\right)$ and, surprisingly, the error term is asymptotically normal, exhibiting an unexpected central limit theorem in St. Petersburg theory.
\end{abstract}

MSC 2010. 60E07, 60F05, 60F17.

Keywords. St. Petersburg sums, semistable process, strong approximation, central limit theorem.

\section{Introduction}

Let $X, X_{1}, X_{2}, \ldots$ be i.i.d. r.v.'s with

$$
P\left(X=2^{k}\right)=2^{-k}, \quad(k=1,2, \ldots)
$$

and let $S_{n}=\sum_{k=1}^{n} X_{k}$. The asymptotic behavior of the sequence $\left\{S_{n}, n \geq 1\right\}$ has attracted considerable attraction in the literature in connection with the St. Petersburg paradox concerning the 'fair' entry fee in a game where the winnings are

*A. Rényi Institute of Mathematics, Reáltanoda u. 13-15, 1053 Budapest, Hungary, e-mail: berkes.istvan@renyi.mta.hu. Research supported by FWF grant P24302-N18 and NFKIH grant K 108615 . 
distributed as $X$. We refer to Csörgö and Simons [9] for the history and bibliography of the problem. Feller [10] proved that

$$
\lim _{n \rightarrow \infty} \frac{S_{n}}{n \log _{2} n}=1 \quad \text { in probability }
$$

(where $\log _{2}$ denotes logarithm with base 2) and Martin-Löf [14] showed

$$
S_{2^{k}} / 2^{k}-k \stackrel{d}{\longrightarrow} G
$$

where $G$ is the infinitely divisible distribution function with characteristic function $\exp (g(t))$, where

$$
g(t)=\sum_{l=-\infty}^{0}\left(e^{i t 2^{l}}-1-i t 2^{l}\right) 2^{-l}+\sum_{l=1}^{\infty}\left(e^{i t 2^{l}}-1\right) 2^{-l} .
$$

Letting $G_{\gamma}$ denote the distribution with characteristic function $\exp \left(\gamma g(t / \gamma)-i t \log _{2} \gamma\right)$ and $\gamma_{n}=n / 2^{\left[\log _{2} n\right]+1}$, Csörgő [6] proved that

$$
\sup _{x}\left|P\left(\frac{S_{n}}{n}-\log _{2} n \leq x\right)-G_{\gamma_{n}}(x)\right| \longrightarrow 0 \quad \text { as } n \rightarrow \infty
$$

and determined the precise convergence rate. In particular,

$$
S_{n_{k}} / n_{k}-\log _{2} n_{k} \stackrel{d}{\longrightarrow} G_{\gamma}
$$

if and only if $\gamma_{n_{k}} \rightarrow \gamma$, a result obtained earlier in [8]. Relation (1.3) shows that the class of subsequential limit distributions of $S_{n} / n-\log _{2} n$ is the class

$$
\mathcal{G}=\left\{G_{\gamma}: 1 / 2 \leq \gamma<1\right\} .
$$

If $n$ runs through the interval $\left[2^{k}, 2^{k+1}\right.$, then $G_{\gamma_{n}}$ moves through the distributions $G_{j / 2^{k+1}}, 2^{k} \leq j \leq 2^{k+1}$ representing, in view of $G_{1}=G_{2}$, a "circular" path in $\mathcal{G}$. In view of (1.3), the distribution of $S_{n} / n-\log _{2} n$ also describes approximately a circular path, a remarkable asymptotic behavior called in [6] merging.

Using a decomposition idea of Le Page, Woodroofe and Zinn [13], in [3] a new representation of the limiting semistable variable of Petersburg sums was given, simplifying the theory considerably and leading to new asymptotic information. Let $\Psi(x)$ denote the function on $(0, \infty)$ which grows linearly from 1 to 2 on any interval $\left[2^{k}, 2^{k+1}\right),(k \in \mathbb{Z})$, let $\eta_{1}, \eta_{2}, \ldots$ be independent exponential random variables with mean 1 and let $Z_{k}=\sum_{j=1}^{k} \eta_{k}$. In [3], Lemma 2 it was proved that for any $1 \leq \gamma<2$ the series

$$
Y^{(\gamma)}=\sum_{j=1}^{\infty}\left[\frac{1}{Z_{j}} \Psi\left(\frac{Z_{j}}{\gamma}\right)-\frac{1}{j} \Psi\left(\frac{j}{\gamma}\right)\right]
$$


converges absolutely with probability 1 and the limit distribution $G_{\gamma}$ above is identical with the distribution of $Y^{(\gamma)}+c_{\gamma}$, where

$$
c_{\gamma}=\sum_{k=1}^{\infty} \frac{\left\{2^{k} \gamma\right\}}{\gamma 2^{k}}-\log _{2} \gamma
$$

Note that (1.5) gives an expansion of the semistable variable $Y^{(\gamma)}$ itself, not of its distribution. A similar decomposition, implicit in [3], holds for the partial sums $S_{n}$, namely

$$
\frac{1}{n} S_{n}-a_{n, \gamma_{n}} \stackrel{d}{=}\left(1+\varepsilon_{n}\right) \sum_{j=1}^{n}\left[\frac{1}{Z_{j}} \Psi\left(\frac{Z_{j}}{\left(1+\varepsilon_{n}\right) \gamma_{n}}\right)-\frac{1}{j} \Psi\left(\frac{j}{\gamma_{n}}\right)\right]+\varepsilon_{n} a_{n, \gamma_{n}}
$$

where $\varepsilon_{n}=Z_{n+1} / n-1, \gamma_{n}=n / 2^{\left[\log _{2} n\right]+1}$ is the dyadic location parameter introduced above and

$$
a_{n, \gamma}=\sum_{j=1}^{n} \frac{\Psi(j / \gamma)}{j}
$$

For the simple proof, see Section 2. Formula (1.6) expands St. Petersburg sums $S_{n}$ in a similar way as the Edgeworth expansions in Csörgö [7], Pap [15] expand the distribution function of $S_{n}$. However, (1.6) provides a pointwise expansion and this makes the formula easier to apply. In particular, (1.6) makes the asymptotic theory of St. Petersburg sums very transparent. By the law of the iterated logarithm we have $\varepsilon_{n}=O\left(n^{-1 / 2}(\log \log n)^{1 / 2}\right)$ a.s. and an easy calculation shows that replacing $\varepsilon_{n}$ by 0 in (1.6) results in an error of $o_{P}(1)$ on the right hand side, and thus we get the result

$$
\frac{1}{n} S_{n}-a_{n, \gamma_{n}}=Y^{\left(\gamma_{n}\right)}+o_{P}(1),
$$

which is meant in the sense that for each fixed $n$ the variables $S_{n}$ and $Y^{\left(\gamma_{n}\right)}$ can be defined on a common probability space such that (1.8) holds. Relation (1.8) thus yields a pointwise version of the merging result (1.3). The purpose of the present paper is to prove that actually much more is valid: the partial sum process of $\left(X_{n}\right)$ can be approximated by a semistable Lévy process $\{L(t), t \geq 0\}$ with $L(1) \stackrel{d}{=} Y^{(1)}$ with a.s. error $O\left(\sqrt{n}(\log n)^{1+\varepsilon}\right)$ and an asymptotically normal error term, establishing an unexpected central limit theorem in St. Petersburg theory.

Theorem 1.1 Let $\{L(t), t \geq 0\}$ be the Lévy process defined by

$$
E(\exp (i u L(t))=\exp (t g(u)) .
$$

where $g$ is the function in (1.2). Then on a suitable probability space one can define the St. Petersburg sequence $\left(X_{n}\right)$ and the process $\{L(t), t \geq 0\}$ jointly such that

$$
\sum_{k=1}^{n} X_{k}=L(n)+O\left(\sqrt{n}(\log n)^{1+\varepsilon}\right) \quad \text { a.s. }
$$


for any $\varepsilon>0$ and

$$
a_{n}^{-1}\left(\sum_{k=1}^{n} X_{k}-L(n)\right) \stackrel{d}{\longrightarrow} N(0,1),
$$

where $a_{n} \asymp(n \log n)^{1 / 2}$.

Here $c_{n} \asymp d_{n}$ means that the ratio $c_{n} / d_{n}$ lies between positive constants. Due to the irregular tail behavior of the random variables in our construction (see the proof of Lemma 2.1), it seems likely that $a_{n} \asymp(n \log n)^{1 / 2}$ in Theorem 1.1 cannot be replaced by $a_{n} \sim c(n \log n)^{1 / 2}$ with a constant $c$.

The process $L(t)$ was introduced by Martin-Löf [14] who proved the scaling relation

$$
g\left(2^{m} t\right)=2^{m}(g(t)-i m t) .
$$

From this it follows that the transformation $t \longrightarrow 2 t$ does not change the distribution of the process

$$
\left\{L(t) / t-\log _{2} t, t>0\right\} .
$$

In particular, $L(2) / 2-1 \stackrel{d}{=} L(1)$, and since $L(2) \stackrel{d}{=} L(1) \star L(1)$, the distribution of $L(1)$ is semistable. In view of the atomic Lévy measure in the characteristic function of $Z(1)$, its distribution is not stable. It also follows that

$$
L(n) / n-\log _{2} n \stackrel{d}{=} L\left(\gamma_{n}\right) / \gamma_{n}-\log _{2} \gamma_{n} \stackrel{d}{=} G_{\gamma_{n}},
$$

showing that $L(n) / n-\log _{2} n$ exhibits the merging behavior (1.3) in an ideal way, with zero error. Thus Theorem 1.1 gives an invariance principle for the merging result (1.3) and actually, for a class of further limit theorems for $\left(X_{n}\right)$. It shows also the surprising fact that the partial sum process of $\left(X_{n}\right)$ can be represented as a semistable Lévy process with an asymptotically normal perturbation.

In a previous paper [1], a strong approximation of St. Petersburg sums with the weaker remainder term $O\left(n^{5 / 6+\varepsilon}\right)$ and without the asymptotic normality of the error term was proved by a standard blocking argument. The proof in [1] works for a large class of i.i.d. sequences $\left(X_{n}\right)$ in the domain of geometric partial attraction of a semistable law $G$. In contrast, the proof of Theorem 1.1 uses the structure of the St. Petersburg sequences in a substantial way and whether Theorem 1.1 remains valid for a larger class of i.i.d. sequences remains open.

Weak and strong approximation of partial sums of i.i.d. random variables $\left(X_{n}\right)$ in the domain of attraction of stable laws were proved in Stout [19], Simons and Stout [18], Berkes and Dehling [2]. The remainder terms there are given in terms of the function $\beta(x)=x^{\alpha}\left|P\left(X_{1}<x\right)-G(x)\right|$, where $G$ is the limit distribution, and are rather complicated. In the case when $\beta(x)$ is a slowly varying function tending to 0 , lower bounds for the remainder term (valid for any construction) are also given in [2], leaving only a small gap between the upper and lower bounds. However, in the case of the stable analogue of St. Petersburg sums, when $G$ is a stable distribution with parameters $\alpha=1, \beta=-1$ (see e.g. [12], p. 164), we have 
$\beta(x)=O\left(x^{-\gamma}\right)$ for some $\gamma>0$ and no lower bounds for the remainder term have been found. For the same reason, we do not have universal lower bounds for the remainder term in the St. Petersburg game and thus, even though Theorem 1.1 determines the precise stochastic order of magnitude of the error term for a specific construction, the question whether other constructions can give a better error term remains open.

\section{Proofs}

We first prove (1.6). Clearly

$$
P\left(X_{1}>x\right)=\Psi(x) / x \quad(x \geq 1) .
$$

Let $F$ denote the distribution function of $X_{1}$ and let $F^{-1}(x)=\inf \{t: F(t) \geq x\}$ be its (generalized) inverse. Then

$$
F^{-1}(x)=2^{k} \quad \text { for } \quad x \in\left(1-2^{-(k-1)}, 1-2^{-k}\right], \quad k=1,2, \ldots
$$

and thus

$$
F^{-1}(1-x)=x^{-1} \Psi(x) \quad \text { for } \quad 0<x<1 .
$$

We also have

$$
\Psi\left(2^{-k} x\right)=\Psi(x) \text { for all } x \in \mathbb{R}, k \in \mathbb{Z} .
$$

As in the Introduction, let $\eta_{1}, \eta_{2}, \ldots$, be independent $\exp (1)$ random variables, $Z_{k}=$ $\sum_{j=1}^{k} \eta_{j}, k=1,2, \ldots$, put

$$
X_{j, n}^{*}=F^{-1}\left(1-\frac{Z_{j}}{Z_{n+1}}\right), \quad 1 \leq j \leq n
$$

and let $X_{1, n} \geq \ldots \geq X_{n, n}$ be the decreasing ordered sample of $X_{1}, \ldots, X_{n}$. By the well known representation of ordered samples (see e.g. [5], page 285 or [17], p. 335), the vector $\left(Z_{1} / Z_{n+1}, \ldots, Z_{n} / Z_{n+1}\right)$ is distributed as the ordered sample $U_{n, 1} \leq \ldots \leq U_{n, n}$ of i.i.d. uniform r.v.'s $U_{1}, \ldots, U_{n}$ in $(0,1)$ and thus the vectors $\left(X_{1, n}, \ldots, X_{n, n}\right)$ and $\left(X_{1, n}^{*}, \ldots, X_{n, n}^{*}\right)$ have the same distribution. By $(2.2)$

$$
\begin{aligned}
X_{j, n}^{*} & =F^{-1}\left(1-\frac{Z_{j}}{Z_{n+1}}\right)=\frac{Z_{n+1}}{Z_{j}} \Psi\left(\frac{Z_{j}}{Z_{n+1}}\right) \\
& =\left(1+\varepsilon_{n}\right) \frac{n}{Z_{j}} \Psi\left(\frac{Z_{j}}{n}\left(1+\varepsilon_{n}\right)^{-1}\right)
\end{aligned}
$$

where $\varepsilon_{n}=Z_{n+1} / n-1$. Now if $2^{k} \leq n<2^{k+1}$, then $\gamma_{n}=n / 2^{k+1}$ and thus from (2.3) we get

$$
\frac{X_{j, n}^{*}}{n}=\left(1+\varepsilon_{n}\right) \frac{1}{Z_{j}} \Psi\left(\frac{Z_{j}}{\gamma_{n} 2^{k+1}}\left(1+\varepsilon_{n}\right)^{-1}\right)=\left(1+\varepsilon_{n}\right) \frac{1}{Z_{j}} \Psi\left(\frac{Z_{j}}{\left(1+\varepsilon_{n}\right) \gamma_{n}}\right)
$$

which implies (1.6) immediately. 
We turn now to the proof of Theorem 1.1, which uses, as in [2], [18], [19], a termwise approximation of partial sums. As it turns out (see Lemma 2.1 below), the termwise error in this approximation is determined by the second term of the expansion (1.5) whose tails were shown in [3] to be $\asymp x^{-2}$. This implies that the termwise error is in the domain of attraction of the normal law, explaining relation (1.11) in Theorem 1.1. The crucial influence of the second term of the expansion (1.5) in our approximation problem is similar to the convergence of Markov chains to the stationary distribution whose speed is determined by the second largest eigenvalue of the transition matrix.

Lemma 2.1 A St. Petersburg variable $X$ with distribution (1.1) and a random variable $Y$ distributed as $Y^{(1)}$ in (1.5) can be jointly defined on a suitable probability space such that

$$
c_{1} x^{-2} \leq P(|X-Y|>x) \leq c_{2} x^{-2} \quad\left(x \geq x_{0}\right)
$$

for some positive constants $c_{1}, c_{2}$.

Proof. Put

$$
W_{1}=\frac{\Psi\left(Z_{1}\right)}{Z_{1}}-1, \quad W_{2}=\frac{\Psi\left(1-e^{-Z_{1}}\right)}{1-e^{-Z_{1}}}, \quad W_{3}=\sum_{k=1}^{\infty}\left(\frac{\Psi\left(Z_{k}\right)}{Z_{k}}-\frac{\Psi(k)}{k}\right) .
$$

We show that (2.5) holds with $X=W_{2}, Y=W_{3}$. Clearly, the distribution function of $Z_{1}$ is $G(x)=1-e^{-x},(x \geq 0)$ and thus $U=G\left(Z_{1}\right)=1-e^{-Z_{1}}$ has distribution $U(0,1)$. Next we observe that for any $k \in \mathbb{Z}$ the function $\Psi(u) / u$ equals $2^{k}$ for $u \in\left[2^{-k}, 2^{-k+1}\right)$ and thus for a fixed $x \in\left[2^{\ell}, 2^{\ell+1}\right), \ell \in \mathbb{Z}$, the inequality $\Psi(u) / u>x$ holds iff $u<2^{-\ell}$. Therefore for $x \in\left[2^{\ell}, 2^{\ell+1}\right)$ we have

$$
P\left(W_{2}>x\right)=P(\Psi(U) / U>x)=P\left(U<2^{-\ell}\right) .
$$

If $x \geq 1$, then $\ell \geq 0$ and thus the last probability in (2.7) equals $2^{-\ell}$; otherwise $\ell<0$ and the last probability in (2.7) equals 1 . Thus $W_{2}$ is a St. Petersburg variable. On the other hand, $W_{3}$ has distribution $Y^{(1)}$ in (1.5) and thus to prove Lemma 2.1 it suffices to show that

$$
c_{1} x^{-2} \leq P\left(\left|W_{2}-W_{3}\right|>x\right) \leq c_{2} x^{-2} \quad\left(x \geq x_{0}\right) .
$$

We first prove that

$$
c_{3} x^{-2} \leq\left(\left|W_{1}-W_{2}\right|>x\right) \leq c_{4} x^{-2} \quad\left(x \geq x_{0}\right)
$$

with some positive constants $c_{3}, c_{4}$. As already noted, for any $k \in \mathbb{Z}$ the function $\Psi(x) / x$ equals $2^{k}$ on the interval $I_{k}=\left[2^{-k}, 2^{-k+1}\right)$. Let now $k \geq 0$ and assume $Z_{1} \in I_{k}$. Then $0<Z_{1}<2,0<1-e^{-Z_{1}}<Z_{1}$ and

$$
1-e^{-Z_{1}}=Z_{1}-\frac{1}{2} Z_{1}^{2}+O\left(Z_{1}^{3}\right)
$$


where the constant in the $O$ is absolute. Thus if $Z_{1} \in I_{k}$, then for $k \geq k_{0}$ we have $1-e^{-Z_{1}} \in I_{k}$ or $1-e^{-Z_{1}} \in I_{k+1}$ and thus

$$
\Delta=\left|W_{1}-W_{2}+1\right|=\left|\frac{\Psi\left(Z_{1}\right)}{Z_{1}}-\frac{\Psi\left(1-e^{-Z_{1}}\right)}{1-e^{-Z_{1}}}\right|
$$

equals 0 or $2^{k+1}-2^{k}=2^{k}$ according as $1-e^{-Z_{1}}$ belongs to $I_{k}$ or $I_{k+1}$ and in view of (2.10) the second alternative can occur only if $Z_{1}$ is closer to the left endpoint of $I_{k}$ than $C 2^{-2 k}$, where $C$ is a constant. But then we have $Z_{1} \sim 2^{-k}, \frac{1}{2} Z_{1}^{2}-O\left(Z_{1}^{3}\right) \sim \frac{1}{2} 2^{-2 k}$ and thus the second alternative occurs iff $Z_{1} \in J_{k}$, where $J_{k}=\left[2^{-k}, 2^{-k}+u_{k}\right)$ with $u_{k} \sim \frac{1}{2} 2^{-2 k}$. Since the density $e^{-x}$ of $Z_{1}$ is $1+O\left(2^{-k}\right)$ on $J_{k}$, we have $P\left(Z_{1} \in J_{k}\right) \sim$ $\frac{1}{2} 2^{-2 k}$ as $k \rightarrow \infty$. We thus proved that the difference $\Delta$ in (2.11) equals $2^{k}$ on a a set $A_{k}$ in the probability space, where the $A_{k}$ are disjoint for $k \geq k_{0}, P\left(A_{k}\right) \sim \frac{1}{2} 2^{-2 k}$ and otherwise $\Delta=0$. Thus

$$
P(\Delta>x) \sim \sum_{2^{k}>x} \frac{1}{2} 2^{-2 k} \sim \frac{2}{3} 4^{-k_{0}} \quad \text { as } x \rightarrow \infty
$$

where $k_{0}$ denotes the smallest integer such that $2^{k_{0}}>x$. This proves (2.9) and we also see that $x^{2} P(\Delta>x)$ and thus $x^{2} P\left(\left|W_{1}-W_{2}\right|>x\right)$ fluctuate between positive constants, without a limit.

Next we observe that

$$
W_{3}-W_{1}=\sum_{k=2}^{\infty}\left(\frac{\Psi\left(Z_{k}\right)}{Z_{k}}-\frac{\Psi(k)}{k}\right)
$$

is a tail sum of the series representing $Y^{(1)}$ in (1.5) whose tail behavior is described by Theorem 5 of [3]; in particular we have

$$
c_{5} x^{-2} \leq P\left(W_{3}-W_{1}>x\right) \leq P\left(\left|W_{3}-W_{1}\right|>x\right) \leq c_{6} x^{-2}
$$

with suitable positive constants $c_{5}, c_{6}$. Theorem 5 of [3] also shows that $x^{2} P\left(\mid W_{1}-\right.$ $W_{3} \mid>x$ ) has no limit as $x \rightarrow \infty$. Now (2.9) and (2.12) imply

$$
P\left(\left|W_{2}-W_{3}\right|>x\right) \leq P\left(\left|W_{2}-W_{1}\right|>x / 2\right)+P\left(\left|W_{1}-W_{3}\right|>x / 2\right) \leq c_{7} x^{-2},
$$

proving the upper half of (2.8). To prove the lower half, we note that

$$
\begin{aligned}
& P\left(\left|W_{2}-W_{3}\right|>x\right) \geq P\left(W_{3}-W_{2}>x\right) \geq P\left(W_{3}-W_{1}>3 x / 2, W_{1}-W_{2}>-x / 2\right) \\
& =P\left(W_{3}-W_{1}>3 x / 2\right)-P\left(W_{3}-W_{1}>3 x / 2, W_{1}-W_{2} \leq-x / 2\right) \\
& \geq P\left(W_{3}-W_{1}>3 x / 2\right)-P\left(W_{3}-W_{1}>3 x / 2,\left|W_{1}-W_{2}\right| \geq x / 2\right) .
\end{aligned}
$$

For any $t \geq 0$, set

$$
V_{t}=\sum_{k=2}^{\infty}\left(\frac{\Psi\left(t+Z_{k}^{*}\right)}{t+Z_{k}^{*}}-\frac{\Psi(k)}{k}\right),
$$


where $Z_{k}^{*}=\sum_{j=2}^{k} \eta_{j}$ for $k \geq 2$. We claim that there exists a positive constant $C$ such that for any $0 \leq t \leq 1$ we have

$$
E\left|V_{t}\right| \leq C
$$

Since the sequence $\left(Z_{k}^{*}\right)$ has the same distribution as $\left(Z_{k}\right)$, for $t=0$ relation $(2.16)$ follows from Lemma 2 of [3]. As inspection shows, the properties of $\left(Z_{k}\right)$ used in the proof in [3] remain valid for the sequence $\left(Z_{k}+t\right)$ for any fixed $t \geq 0$ and moreover, the inequalities in [3] hold uniformly for $0 \leq t \leq 1$, proving (2.16). Now, conditionally on $Z_{1}=t, W_{3}-W_{1}$ becomes $V_{t}$ in (2.15) which is independent of $\eta_{1}=Z_{1}$ and thus of $\Delta=\left|W_{1}-W_{2}+1\right|$ in (2.11) and consequently for $x \geq x_{0}$

$$
\begin{aligned}
& P\left(W_{3}-W_{1}>3 x / 2,\left|W_{1}-W_{2}\right| \geq x / 2 \mid Z_{1}=t\right) \\
& \leq P\left(W_{3}-W_{1}>3 x / 2, \Delta \geq x / 4 \mid Z_{1}=t\right) \\
& =P\left(V_{t}>3 x / 2\right) I\{\Delta(t) \geq x / 4\} \\
& \leq \frac{2}{3 x} E\left|V_{t}\right| I\{\Delta(t) \geq x / 4\} \leq C x^{-1} I\{\Delta(t) \geq x / 4\},
\end{aligned}
$$

where $\Delta(t)$ is the expression in (2.11) with $Z_{1}$ replaced by $t$. If $Z_{1}$ is bounded away from 0 , then $\left|W_{1}-W_{2}\right|$ is bounded above, or putting differently, if $\left|W_{1}-W_{2}\right|$ is large, then $Z_{1}$ is near 0 . Thus integrating $(2.17)$ over $0 \leq t \leq 1$ with respect to $P\left(Z_{1} \in d t\right)$ we get

$$
P\left(W_{3}-W_{1}>3 x / 2,\left|W_{1}-W_{2}\right| \geq x / 2\right) \leq C x^{-1} P(|\Delta|>x / 2) \leq C^{\prime} x^{-3}
$$

for sufficiently large $x$, where in the last step we used (2.9). Now using (2.12), (2.14) and (2.18) we get the lower half of (2.8).

Proof of Theorem 1.1. For the vector $(X, Y)$ in Lemma 2.1, let $H$ denote the distribution function of $X-Y$ and put

$$
U(x)=\int_{|t| \leq x} t^{2} d H(t) .
$$

Using Lemma 2.1 and integration by parts we get

$$
\begin{aligned}
& U(x)=-x^{2}(1-H(x)+H(-x))+\int_{0}^{x} 2 t(1-H(t)+H(-t)) d t \\
& =O(1)+\int_{0}^{x} 2 t(1-H(t)+H(-t)) d t
\end{aligned}
$$

provided that $x$ and $-x$ are continuity points of $H$. Using Lemma 2.1 again for the last integral it follows that

$$
c_{8} \log x \leq U(x) \leq c_{9} \log x \quad \text { and } \quad U(2 x)-U(x)=O(1) \quad \text { as } x \rightarrow \infty
$$

with suitable positive constants $c_{8}$ and $c_{9}$. Thus $\lim _{x \rightarrow \infty} U(2 x) / U(x)=1$, i.e. the nondecreasing function $U$ is slowly varying. Further, (2.5) implies that $H$ has a finite 
expectation. Let now $\left(X_{n}, Y_{n}\right)$ be i.i.d. copies of the vector $(X, Y)$ in Lemma 2.1. By the slow variation of $U, X-Y$ is in the domain of attraction of the normal law, specifically we have

$$
\frac{1}{a_{n}} \sum_{k=1}^{n}\left(X_{k}-Y_{k}-c\right) \stackrel{d}{\longrightarrow} N(0,1)
$$

where $c=E(X-Y)$ and the norming sequence $\left(a_{n}\right)$ satisfies

$$
a_{n}^{2} \sim n U\left(a_{n}\right) \quad \text { as } \quad n \rightarrow \infty .
$$

(See e.g. [11], p. 580, Theorem 3.) Using (2.22) and the first relation of (2.20), we get by a simple calculation

$$
c_{10}(n \log n)^{1 / 2} \leq a_{n} \leq c_{11}(n \log n)^{1 / 2}
$$

with suitable constants $c_{10}, c_{11}$. Recall now that along the sequence $n=2^{k}$ we have

$$
\frac{1}{n} \sum_{k=1}^{n} X_{k}-\log _{2} n \stackrel{d}{\longrightarrow} G, \quad \frac{1}{n} \sum_{k=1}^{n} Y_{k}-\log _{2} n \stackrel{d}{\longrightarrow} G
$$

where $G=G_{1 / 2}$ is the semistable distribution defined after (1.2). The first relation here follows from (1.4) and the second from (1.13), since $\sum_{k=1}^{n} Y_{k} \stackrel{d}{=} L(n)$. Relation (2.23) shows that replacing $1 / a_{n}$ by $1 / n$ in (2.21), the left hand side will converge to 0 in probability and adding the second relation of (2.24) yields

$$
\frac{1}{n} \sum_{k=1}^{n} X_{k}-\log _{2} n-c \stackrel{d}{\longrightarrow} G
$$

which, together with the first relation of (2.24), implies $c=0$ and thus (2.21) yields

$$
\frac{1}{a_{n}} \sum_{k=1}^{n}\left(X_{k}-Y_{k}\right) \stackrel{d}{\longrightarrow} N(0,1) .
$$

Since the process $\left\{\sum_{k=1}^{n} Y_{k}, n \geq 1\right\}$ has the same distribution as $\{L(n), n \geq 1\}$ where $L$ is the Lévy process defined by (1.9), relation (1.11) is proven.

To prove (1.10), let $b_{n}=\sqrt{n}(\log n)^{1+\varepsilon}, \varepsilon>0$. Then using Lemma 2.1, (2.20) and integration by parts one can easily verify the relations

$$
\sum_{n=1}^{\infty} \frac{1}{b_{n}^{2}} \int_{|x|<b_{n}} x^{2} d H(x)<+\infty, \quad \frac{1}{b_{n}} \sum_{k=1}^{n} \int_{|x|<b_{n}} x d H(x) \longrightarrow 0
$$

and

$$
\sum_{n=1}^{\infty} P\left(|X-Y| \geq b_{n}\right)<+\infty
$$


(In the case of the second relation of (2.26) replace the domain $\left\{|x|<b_{n}\right\}$ of integration by $\left\{|x| \geq b_{n}\right\}$ in view of $E(X-Y)=0$.) Thus using Theorem 6.8 in Petrov [16], p. 211 we get

$$
\frac{1}{b_{n}} \sum_{k=1}^{n}\left(X_{k}-Y_{k}\right) \longrightarrow 0 \text { a.s., }
$$

yielding (1.10).

\section{References}

[1] Berkes, I.: Strong approximation of the St. Petersburg game. Statistics, to appear.

[2] Berkes, I. and Dehling, H.: Almost sure and weak invariance principles for random variables attracted by a stable law. Prob. Theory Rel. Fields 83 (1989), $331-353$.

[3] Berkes, I., Győrfi, L. and Kevei, P.: Tail probabilities of St. Petersburg sums, trimmed sums, and their limit. J. Theor. Probability, to appear.

[4] Bernoulli, D.: Specimen theoriae novae de mensura sortis. Comm. Acad. Sci. Imp. Petropolitanae 5 (1738), 175-192. German translation: A. Pringsheim: Versuch einer neuen Theorie der Wertbestimmung von Glücksfällen, Verlag von Duncker \& Humblot, Leipzig, 1896.

[5] Breiman, L.: Probability. Second edition, SIAM, 1992.

[6] Csörgö, S. Rates of merge in generalized St. Petersburg games. Acta Sci. Math. (Szeged) 68 (2002), 815-847.

[7] Csörgö, S.: Merging asymptotic expansions in generalized St. Petersburg games. Acta Sci. Math. (Szeged) 73 (2007), 297-331.

[8] Csörgö, S. and Dodunekova, R.: Limit theorems for the Petersburg game, in: Trimmed sums and extremes (M. G. Hahn, D. M. Mason, D. C. Weiner, eds.) pp. 285-315. Progress in Probability, Vol. 23, Birkhäuser, Boston 1991.

[9] Csörgö, S., Simons, G.: A bibliography of the St. Petersburg paradox. University of Szeged, 2014.

[10] Feller, W.: Note on the law of large numbers and "fair" games. Ann. Math. Statist. 16 (1945), 301-304.

[11] Feller, W.: An introduction to probability theory and its applications, Vol II, second edition. Wiley, 1971.

[12] Gnedenko, B.V. and Kolmogorov, A.N.: Limit distributions of sums of independent random variables. Addison-Wesley, 1954

[13] LePage, R., Woodroofe, M. and Zinn, J.: Convergence to a stable distribution via order statistics. Ann. Probab. 9 (1981), 624-632. 
[14] Martin-Löf, A.: A limit theorem which clarifies the 'Petersburg paradox'. J. Appl. Probab. 22 (1985), 634-643.

[15] Pap, Gy.: The accuracy of merging approximation in generalized St. Petersburg games. J. Theoret. Probab. 24 (2011), 240-270.

[16] Petrov, V.V.: Limit theorems of probability theory. Oxford University Press, 1995.

[17] Shorack, G. and Wellner, J.: Empirical processes with applications to statistics. Wiley, 1986.

[18] Simons, G. and Stout, W.: A weak invariance principle with applications to domains of attraction. Ann. Probab. 6 (1978), 294-315.

[19] Stout, W.F: Almost sure invariance principles when $E X_{1}^{2}=\infty$. Z. Wahrsch. Verw. Gebiete 49 (1979), 23-32. 